Médée; figure féminine et mythique de l'antiquité au temps contemporain

\author{
DR. MONA ELKAYYAL \\ MAÎTRE DE CONFÉRENCES EN LITTÉRATURE \\ FRANÇAISE \\ Université de Mansourah \\ Faculté de pédagogie
}




\section{INTRODUCION}

De l'antiquité à nos jours, le mythe a constitué une entreprise complexe. C'est la cause pour laquelle les littéraires, les sociologues et les anthropologues ne cessent de l'analyser par les manières les plus divers. Selon Mircéa Eliade:

"C'est le mythe qui raconte des histoires sacrées, il relate un événement qui s'est passé au temps primordial, au temps fabuleux du commencement [...]. II raconte comment, grâce aux exploits des êtres surnaturels, une réalité est venue à l'existence. ${ }^{1}{ }^{1}$

Le mythe permet à l'imaginaire humain de répondre à la question de ses origines: la mort ou la part du destin. Les dramaturges du XXème siècle envisagent les relations de l'homme avec les forces de l'univers.

«[...] Le mythe peut répondre aux besoins fondamentaux de la pensée humaine .[...] Tout ce qui n'est pas éclairé par l'esprit rationnel appartient au mythe. " ${ }^{2}$

\section{Qu'est-ce qu'un mythe littéraire ?}

Le mythe littéraire est considéré comme une histoire qui explique et fonde les pratiques sociales. Le mythe est différent de la légende (repose sur des faits historiques identifiables), l'histoire (invention sans expliquer), et le roman (explication avec un peu de base.) ${ }^{3}$ Le mythe a une vérité symbolique : elle donne au monde et aux relations humaines, un sens qu'elle ne peut démontrer. Pierre Grimal explique :

1 - Mircea Eliade, Aspect du mythe, Paris, Gallimard, 1966, pp. 16-17.

2 - Pierre Grimal, Dictionnaire de la mythologie grecque et romaine, Paris, PUF, 1951. p. 12.

\section{3- https://litterature.savoir.fr/mythe-litteraire-definition}


Les mythes sont inséparables de toutes pensées, dont ils forment un élément vital. Elle essaye de les mieux connaître, c'est pénétrer dans la pensée des hommes.) ${ }^{1}$

En effet, le mythe a présenté depuis l'antiquité jusqu'à nos jours, quelques grandes figures, parmi lesquelles on peut trouver des récits d'une féminité redoutable et nocturne. Médée nous donne un exemple qui incarne une fatalité inquiétante.

\section{PROBLÉMATIQUE DE L'ÉTUDE}

Médée, une de ces grandes figures mythiques plusieurs fois, ont été traduites en littérature. Personnage héroïque, considéré comme l'une des créatures féminines les plus célèbres dans la mythologie antique.

L'histoire de cette magicienne et meurtrière de ses enfants est l'image effrayante de l'infanticide que les auteurs offrent à travers différentes représentations que celle d'Euripide*. A l'instar $d^{\prime}$ 'Euripide les dramaturges s'accordent à la représenter sous l'image du Mal: Médée incarne en elle une certaine ambiguïté parce que, dans son désarroi amoureux, elle n'a trouvé pour moyen que se venger. Exilée, magicienne, meurtrière passionnée, Médée se présente comme une figure transgressive.

\section{Depuis le XVlème siècle, le mythe de Médée continue sans cesse d'évoluer.}

\section{Pourquoi une étude sur Médée?}

Passant le temps grec et latin, Médée a une différente dimension. Il est évident que, les autres dramaturges, au XIXè et au XXè siècle, s'inscrivent dans une vision moderne. Ces

1- Pierre Grimal, op, cit, p.12.

*Euripide a été le premier dramaturge qui présente Médée sous une forme humaine. Dépouillée de son statut divin, Médée est une femme outragée, blessée. C'est une femme qui souffre parce que seule et abandonnée. La souffrance l'emporte sur tout le reste, elle songe à mourir et ne supporte plus la vue de ses enfants. 
reprises modernes reflètent l'évolution de la société, de la vision de la femme et de la condition féminine, nous remarquons un changement de point de vue sur le mythe de Médée. (Une Médée moderne).

\section{Une histoire non plus antique mais moderne}

A l'inverse du mythe traditionnel, Médée est présentée plutôt comme une victime dans une société où la femme est marginalisée. Médée échappe donc à la vision traditionnelle à un autre statut, celui de la femme victime d'une société injuste.

Certains dramaturges tels Jean Anouilh et Henny Hans Jahnn nous donnent une vision différente de Médée qui est considérée comme un personnage fatal. Ces auteurs restent tous fidèles à l'esprit d'Euripide, mais introduisent des variantes en s'écartant de la mère infanticide:

"La problématique de la femme amoureuse, bafouée, demeure l'assise de leur transposition bien qu'ils aient modifié la psychologie de leurs personnages.. ${ }^{1}$

Les dramaturges contemporains ont changé le point de vue ainsi que le cadre historique, géographique et le statut social des personnages tout en préservant pour l'essentiel sa structure.

\section{CORPUS DE L'ÉTUDE}

Nous avons porté notre choix sur des œuvres couronnées de succès: Médée, pièce de théâtre de l'Allemand Hans Henny Jahnn $(1926)^{3}$. Chez lui, Médée est une princesse noire dont le fils aîné, est le rival malheureux de son père Jason. Les deux sont déchirés par l'amour qu'ils vouent à Créüse.

\footnotetext{
${ }^{1}$-Alain Depaulis, Le Complexe de Médée : quand une mère prive le père de ses enfants, Bruxelles, De Boeck et Larcier s. a. 2003, p. 53.

2- Hans Henny Jahnn, Médée [1963], Paris, José Corti, 1988. Jahnn Hans Henny, Médée, traduit par Huguette et René Radrizzani, Paris, éd. José Corti, 1998.
} 
Médée, pièce de théâtre de Jean Anouilh $(1946)^{1}$. Anouilh y a adapté le mythe antique de la magicienne, la princesse, petite-fille du Soleil. En plus, nous avons choisi ces auteurs et leurs œuvres, parce qu'ils sont tous issus de cultures différentes, Médée, transposé dans une époque contemporaine. Tous font de Médée une étrangère exilée dans " la moderne Corinthe. " ${ }^{2}$

\title{
MÉTHODE D'ANALYSE
}

Pour les besoins de notre recherche, il s'agira de faire appel à la méthode comparatiste : elle nous permettra d'établir des liens entre les différentes écritures, dégager les parentés et les divergences entre elles. En effet, la perspective de la littérature comparée est très importante dans notre recherche comme le soulignent $P$. Brunel, C. Pichois et André-Michel Rousseau :

\begin{abstract}
«La littérature comparée est l'art méthodique qui recherche les liens d'analogie, d'influence. Elle rapproche la littérature des autres domaines de l'expression ou de la connaissance qui appartiennent à plusieurs langues ou plusieurs cultures. Afin de mieux les décrire, les comprendre et les goûter.. ${ }^{3}$
\end{abstract}

\section{CHAPITRE I: SOURCES ET RÉSUME DU MYTHE DE MÉDÉÉ}

Le mythe de Médée s'inscrit dans le cycle des Argonautes. C'est surtout grâce à Euripide (v. 480-406 av. J.-C.) que ce mythe a été passé à la postérité. Après Euripide, plusieurs auteurs se sont penchés sur la figure de Médée. Euripide a été le premier à reprendre le mythe de Médée au théâtre. La pièce est jouée en 431 avant J.-C. Elle expose essentiellement la différence entre Jason et Médée.

\footnotetext{
${ }^{1}$-Jean Anouilh, Médée, Éditions de la Table ronde, 1947. Création de la pièce : 1946.

2- Duarte Mimoso Ruiz, Avatars modernes du mythe antique dans deux Médée Africaines, Strasbourg : 1980, p. 247.

3- Pierre Brunel, Claude Pichois, André-Michel Rousseau, Qu'est-ce que la littérature comparée? Paris : Armand Colin, 1996, p. 150.
} 
Dans son prologue, l'auteur ne fait qu'un bref rappel de la mission des Argonautes. Ce qui l'intéresse, ce sont les sentiments de douleur qu'éprouve Médée face à l'infidélité de Jason.

Selon Alain Moreau :

" Euripide est donc l'écrivain qui a infléchi le mythe et fait de Médée cette magnifique et redoutable figure qui s'est dès lors imposée dans la littérature ${ }^{1}$.

Pour Euripide, Médée est victime d'une injustice et c'est surtout le fait que Jason l'a abandonnée qui explique son crime.

Seule la Médée d’Euripide est restée dans son intégralité.

Le mythe de Médée comporte cinq épisodes. Dans le premier, Médée aide Jason dans sa quête de la Toison d'or. Le deuxième épisode se situe à lolcos, en Thessalie. Jason rapporte la Toison d'or à son oncle Pélias. Jason et Médée ont vécu en bons termes avec Pélias.

Le troisième, centré sur la mort des enfants de Médée et Jason. L'action se situe à Corinthe. On y raconte l'abandon de Médée par Jason, qui veut épouser Créüse. Condamnée à l'exil, la jeune princesse meurt dès qu'elle la revêt. Toujours pour se venger de Jason, Médée décide aussi de tuer ses propres enfants puis elle s'enfuit dans un char envoyé par le Soleil. Dans le quatrième épisode, Médée se réfugie à Athènes et épouse le roi Égée . Lorsque Thésée revient chez lui afin de se faire reconnaître par son père, Médée cherche à le faire disparaître. Égée comprend toutefois que Thésée est son fils et non un ennemi comme le lui faisait croire Médée.

En conséquence, le roi la répudie et l'exile. C'est alors que Médée, et là se situe le cinquième et dernière épisode, retourne en Colchide. Elle aide son père Aeétès à reprendre son trône.

La problématique générale de la Médée d'Euripide concerne le différend entre Médée et Jason. Euripide expose les

1 -Moreau, Alain, « Médée », Dictionnaire des mythes féminins, sous la direction de Pierre Brunei, Monaco, Du Rocher, 2002, p. 1282. 
sentiments qu'éprouve Médée face à l'infidélité de Jason et le tiraillement de la jeune femme entre son amour pour ses enfants et son désir de vengeance.

\section{Le personnage de Médée chez Euripide}

\section{Amoureuse : Couple formé avec Jason}

Dès la première page de la pièce, Elle tombe amoureuse du grec Jason venu en Colchide chercher la Toison d'Or. Toujours est-il que Médée aide le bel étranger à vaincre les épreuves imposées par le roi pour conquérir la Toison. Elle décide de fuir son pays à bord de l'Argos qui fait voile vers la Grèce. A-telle d'autre choix que de suivre l'homme qu'elle aime puisqu'elle a trahi son père et son pays ?

2. Trompée et folle de rage : Jason abandonne Médée pour une autre femme

En dépit de ces sacrifices, qui sont autant de preuves d'amour, " traître à ses enfants et à sa maîtresse, Jason est entré par l'hymen dans une couche royale, en épousant la fille de Créon (Créuse), l'arbitre souverain du pays " (p. 123). 1

Face à l'infidélité de Jason, Médée éprouve une profonde souffrance : " Elle gît sans nourriture, abandonnant son corps aux chagrins, consumant tous ses jours dans les pleurs ) « [...]p. 124).

Plus loin, Médée révèle qu'elle a même tué son propre frère pour servir les desseins de Jason. Sa souffrance est telle que tout sentiment maternel semble l'avoir désertée : "Ses enfants lui font horreur, elle n'a plus de joie à les voir " (p. 124).

${ }^{1}$-Euripide, Médée, Paris, Les Belles lettres, 1965.

2- Duarte Mimoso-Ruiz, Médée antique et moderne, Aspects rituels et sociopolitiques d'un mythe, op. cit., p. 152.

3- http://www.mythologie.ca/heros/medee.html 
Il est évident que la Médée d'Euripide propose une réflexion sur les relations amoureuses. La difficile harmonie entre I'homme et la femme. C'est l'opposition entre raison et passion :

" La tragédie a pour centre, précisément, ce manque d'harmonie entre raison et passion, et la désagrégation de la famille à la suite de ce désaccord fondamentalı ${ }^{2}$

Folle de jalousie et de douleur, Médée tue la fiancée de son mari, puis ses deux garçons avant d'incendier Corinthe et de s'enfuir. Estelle vraiment devenue une infanticide?

" Elle est magicienne, sorcière, elle connaît les secrets de la vie et de la mort. Pour tout cela, elle agit comme un homme, elle refuse de subir et de se laisser faire.. ${ }^{3}$

Euripide peint une Médée déchirée entre son amour maternel pour ses enfants et sa soif de vengeance envers l'amant qui l'a bafouée.

\section{Jason la qualifie de monstre}

\section{Magicienne}

Médée est une puissante magicienne. C'est par la magie qu'elle a aidé Jason en Colchide, c'est par la magie qu'elle fait tuer Pélias par ses propres filles.

Donc, on peut dire que Médée d'Euripide nous présente deux images contradictoires : d'une part, il y a la femme trahie par l'homme pour lequel elle a tout sacrifié, d'autre part, Euripide la représente comme étant capable des pires crimes, dont celui de tuer ses propres enfants.

Médée nous apparaît donc comme un être torturé par son amour et par sa haine envers sa famille : 
" Euripide a été le premier dramaturge qui présente Médée sous une forme humaine. Dépouillée de son statut divin.» 1

Médée à toutes les sauces, innocente, cruelle, démoniaque, libre,

" Médée, monstrueuse, solide, féminine, bafouée, mère dénaturée ou bouc-émissaire, est, quel que soit le qualificatif que le lecteur lui donne et la manière dont il la voit, un personnage tragique, complexe et fascinant, source d'inspiration et de réflexion intarissable. ${ }^{2}$

Médée est une figure importante de la mythologie grecque et I'héroïne d'une liste d'œuvres longue comme un bras depuis l'antiquité jusqu'à aujourd'hui.

\section{CHAPITRE II: MÉDÉE}

Hans Henny Jahnn

Médée, le chef-d'œuvre dramatique de Jahnn a été écrit pendant l'aboutissement de la période de jeunesse. Jahnn renouvelle le nœud, le déroulement du drame, la thématique et la description des personnages.

Des oppositions non seulement femme / I'homme, mais aussi entre la jeunesse et la vieillesse, la sexualité et la mort et la lumière et l'obscurité :

"Médée, femme bafouée, tenue, loin d' aucune civilisation patriarcale, plus proche de la nature. C'est une barbare : ses fils sont des mulâtres, méprisés par les blancs civilisés. ${ }^{2}$

\footnotetext{
${ }^{1}$ - Koua, Viviane, Médée figure contemporaine de l'intercu/turalité (Thèse de doctorat) - Université de Limoges et Université de Cocody, op. cit., p. 9.

2-https://www.etudes-litteraires.com/forum/topic44268-max-rouquette-medeescene-21.html

${ }^{3}$-https://www.chapitre.com/BOOK/jahnn-hans-

henny/medee,1203491.aspx\#product-details-and-summary
} 


\section{Hans Henny Jahnn:}

Hans Henny Jahnn, né le 17 décembre 1894 à HambourgStellingen et mort le 29 novembre 1959 à Hambourg, est un romancier, dramaturge, facteur d'orgue et éditeur de musique allemand.

Médée de Jahnn relit le mythe sous l'angle sensuel et racial.

Quand Jahnn écrit sa Médée (1925), le sujet racial est très présent dans l'actualité politique. Jahnn fait de son héroïne un africaine dont les fils sont des métis, méprisés par les blancs civilisés.

Jahnn, poète révolté (contre toutes les formes d'inégalité et d'injustice.) Médée est la figure de l'étrangère de tous les pays. L'univers de Jahnn est d'une sensualité profonde. Toute son œuvre est hantée par ses propres obsessions: la décomposition de la chair, la sexualité, la mort...

" Dans cette œuvre, chaque phrase est pleine de grandes hésitations pour être juste au point de vue du rythme et du contenu. II y a des phrases ont poussé Jahnn au bord du suicide. " ${ }^{1}$

\section{Hans Henny Jahnn.}

Inspirée d'Euripide, son mythe est au centre d'une pluralité de tragiques : tragique des enfants de Médée, tragique de Jason, sans statut social, tragique de Médée, la «barbare», la noire, qui porte la couleur de "l'autre» et de toutes les

1- http://www.fabriktheatre.com/spectacles/medee-de-hans- henny- Jahnn/

2- Hans Henny Jahnn, Texte français René Radrizzani, Extrait de «Les Légendes de Médée et de savie" "1927.

3-Médée de Hans Henny Jahnn traduit par Huguette et René Radrizzani , José Corti , 1988 pour l'édition allemande, 1926 pour la première version, 1998 pour la présente traduction (https://femmes-de-lettres.com/2015/02/21/les-reecrituresde-medee-medee-de-hans-henny-jahnn/)

4- Ibid. 
exclusions. L'acte de Médée prend une nouvelle signification, une signification politique et sociale de condamnation d'une société injuste et inhumaine:

"Nous tous, êtres humains, avons traversé I'humanité en portant tous les marques des illuminations et des asservissements cruels. Elles n'appartiennent pas au passé, malgré l'évolution des coutumes des Européens. ${ }^{2}$

" Médée, c'est moi ${ }^{3}$ Hans Jenny Jahnn se sentait luimême "femme, marginal, barbare", et a pu "réinventer le mythe de l'intérieur " ${ }^{4}$.

La nouveauté de cette Médée au XXe siècle réside dans quelques transformations : dans le nœud, le déroulement, la thématique et les personnages : Médée est devenue noire, et ses fils sont parvenus à l'adolescence.

Dans la tragédie antique, Jason, Créüse et Médée avaient un certain prestige (de noblesse et d'héroïsme). La conception des personnages est vouée à la banalisation et la dégradation morale, physique et sociale. Jason, d'un personnage auréolé de gloire à un personnage ordinaire. Il est déchu de son rang mythique:

«Des relations incestueuses existent dans la famille, le père a des relations sexuelles avec le fils aîné et le fils aîné avec le frère, ce que Jahnn nomme pudiquement " être ami ». ${ }^{1}$

${ }^{1}$-https://femmes-de-lettres.com/2015/02/21/les-reecritures-de-medee-medeede-hans-henny-jahnn/

2- Hans Henny Jahnn, Médée [1963], Paris, José Corti, 1998, p. 14.

3- Ibd., p. 15.

4- http://next.liberation.fr/livres/1998/10/29/espece-de-medee-une-medee-de1926-violente-et-raciale-par-hans-henny-jahnn-allemand-a-la-sensualite-_249333 
Jahnn le présente comme un personnage pervers à cause de ses appétits sexuels désordonnés. Ce comportement incestueux du père éloigne les deux frères. Le fils cadet:

« II ne m'aime pas parce que tu es son ami.» ${ }^{1}$

Le fils aîné rétorque :

"Je t'aime et père t'aimera autant que moi et sera ton ami, lorsque tu seras éveillé, pleinement toi-même.» ${ }^{3}$

Une très grande vitalité sexuelle se trouve dans l'œuvre de Jahnn et Médée, attend l'amour de Jason qui ne vient plus et aussi, il va épouser une autre. La sexualité joue un rôle central dans cette version. L'homosexualité de Jason, pratiquée à l'égard de son fils et de ses domestiques. Cette pratique explique le fait que:

" La maison est pleine de beaux hommes, mais pas de femmes; c'est pourquoi on n'entend pas de cris d'enfants, ne voit point de fruit bomber des ventres. " ${ }^{4}$

La sexualité fait de Jason, un époux infidèle. II n'arrive plus à contrôler ses instincts. Médée considère Jason ici, un obsédé sexuel.

En effet, le personnage de Médée a perdu chez Hans Henny son statut de princesse à une simple mère. Médée n'est plus cette princesse, mais radieuse, sublime, séduisante est transformée en femme vieillissante abandonnée par son mari:

"Une vie périlleuse, et la déchéance de l'éternelle beauté. A une grecque, tu n'oserais pas dire les insultes que, sans hésiter, tu m'adresses. Une barbare, dis-tu en parlant de ma peau sombre. ${ }^{1}$

1. Hans Henny Jahnn, Médée, op.cit. p. 25. 
Mais Jason subit un destin encore terrible, comme celui de I'héroïne: à cause de la sexualité qui se présente comme une force, une violence, une injustice.

Médée sacrifie sa jeunesse au profit de ses fils et de Jason son époux. Proche de la tombe، le corps de la Médée qui était si beau ne rayonne plus chez Jahnn. Elle n'hésite pas à souligner à Jason combien de fois elle a dû vieillir à cause de lui :

«[...]Voici devant vous une femme grasse, grise, noirâtre, voici mes seins, gras et flasques à la fois, le pied bot est assorti à la graisse. Voulez-vous me voir danser, comme tout sur moi ballotte? ${ }^{1}$

Jason restera éternellement jeune ainsi que leurs fils.

La magie de Médée ne s'arrête pas uniquement à l'action bienfaitrice et sacrificatoire. Ainsi Médée la magicienne se transforme en une infanticide lorsqu'elle voit ses intérêts menacés.

Apprenant le mariage de Jason avec Créüse, Médée ne sait que faire. La seule façon est la vengeance. Elle décide de commettre des crimes irréparables par des forces naturelles.

Le mythe de Médée chez les écrivains antiques comme chez les modernes, commence et se termine sur une scène de violence.

La mort d'Absyrte (son frère) est tragique. Médée pour échapper aux soldats de son père qui les poursuivaient dans leur fuite vers la Corinthe, tue son frère (le petit fils du soleil) à l'aide d'une hache:

«[...]Elle lui arrachait le cœur. Et le jeta dans la mer verte, qui alors se transmua en sang et

1- Ibid. p. 97. 
s'apaisa. Elle coupa ses mains et jeta les morceaux de son corps dans la mer rouge, coagulée. ${ }^{1}$

II y a aussi des faits tragiques tels que l'aveuglement sanglant du messager venu annoncer le mariage de Jason et Créüse:

«A présent tu désignes tes yeux, ces menteurs, comme coupables. Arrachez-les-lui ! Même s'ils annonçaient la vérité. Celui qui voit l'adultère de Jason doit devenir aveugle. $\nu^{2}$

Créüse est, dans cette même version, une fille perverse. Elle est d'abord l'amante du fils de Jason, puis tombée amoureuse du père. On voit comment père et fils sont indifféremment partenaires sexuels pour Créüse.

Créüse, une fille douce et innocente devient chez Jahnn, un personnage sulfureux.

Réception du destin ou de la tragédie personnelle de Médée

Nous voulons porter une attention particulière au personnage de Médée: son être à travers son faire, les différents motifs qui la caractérisent dans l'ouvre de Hans.

Pourquoi l'être de Médée se mue pour se conjuguer à l'universel.

1. Médée : De la passion à la vengeance

Comment cette passion, éprouvée pour Jason, s'est révélée et comment elle a fini par devenir une passion meurtrière?

Dans le mythe antique, il est dit que la déesse Héra, demanda à Aphrodite, déesse de l'amour, d'aider Jason afin que Médée

1- Ibid, p. 74.

2- Ibid, pp. 47-48. 
tombe éperdument amoureuse de lui. Médée est désormais prête à partir avec lui et devenir sa femme.

Chez Jahnn, par contre, Médée tombe éperdument amoureuse de lui. La première rencontre se passant dans le temple. En épousant Jason, elle met à son service son pouvoir surnaturel.

Découvrant la trahison de Jason, elle décide de se venger afin de détruire tout ce qu'il aime. Elle commet des actions fatales et désastreuses. Médée tue son frère et parvenue à tuer sa rivale. Chez Jahnn, comme chez Euripide, Créüse brûle en effet sous l'action du feu occasionné par Médée.

L'indifférence de Jason face à sa passion, à sa sensualité sera à la base de la séparation du couple où se mêlent sensualité et sexualité.

\section{La sensualité et la sexualité}

La sensualité et la sexualité jouent un rôle prépondérant dans Médée modern de Hans. En effet, avec ce dramaturge, Jason vit une sexualité affirmée. Il s'agit de la relation qu'il entretient avec des hommes (homosexualité). Jason affiche les relations sexuelles qu'il vit avec son fils aîné. Bien plus encore, Jahnn oppose le père et le fils qui aiment une même femme: Créüse.

Contrairement à Jason qui est jeune, Médée vieillit et voit sa beauté se faner. Malgré cette vieillesse, elle ressent toujours la sensualité en elle. Elle éprouve toujours du désir pour son mari. Médée considère l'abandon de Jason comme une insulte à sa féminité. Elle lui reproche de négliger sa couche.

Malgré son abandon, Médée garde toujours l'espoir d'accompagner son fils aîné pendant sa nuit de noce. Cependant, cette joie ne viendra jamais. Jason, au lieu de demander à Créon la possibilité pour son fils d'épouser Créüse, c'est lui qui l'épousera. Médée dira à Jason :

«Tu as trompé ton propre fils, tu lui as volé sa fiancée, tu me refuses, à moi, vieille femme, le 
récompense qui me revient pour l'avoir mis au monde. " ${ }^{1}$

Finalement, elle sera privée de sa vie de femme, de mère et d'épouse.

Aussi ses enfants entretiennent des rapports charnels. Jahnn apparaît qu'homosexualité et inceste sont des pratiques courantes chez Jason et ses enfants. Jahnn met plus l'accent sur la sensualité; malgré sa vieillesse, Médée peut toujours éprouver du désir sexuel jusqu'au jour où Jason lui déclare la cause de son rejet :

«Femme! Je romps notre union. Tu as commis des crimes par milliers. Ensorcelé tout ce qui

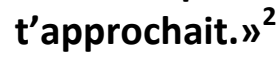

Sous le poids de la jalousie, Médée éprouve une haine contre Jason et la nouvelle compagne.

\section{L'infanticide}

Médée tue ses enfants dans la fierté. Certes, il s'agit de punir Jason de son infidélité mais la raison essentielle est le fait qu'elle ne souhaite pas que les Corinthiens les tuent. Jason devait payer par cette mort parce qu'il s'est volontairement séparé de sa famille. En effet, après la blessure causée par leur père, les enfants sont heureux de se retrouver enfin unis à jamais. Médée qui les trouve dans une relation incestueuse, ne peut résister à cette scène :

«Pleurant au sol, haletant, se roulant l'un sur l'autre. C'était comme une noce. " ${ }^{2}$

1. Hans Henny Jahnn, op.cit. p. 94.

1. Ibid. p. 76.

2. Ibid. p. 95. 
Son meurtre trouve un sens nouveau bien que la vengeance. Elle dit refuser de voir ses enfants se déchirer pour l'amour de leur père. Elle a décidé de les unir dans la mort. A cet effet, Mimoso Ruiz constate un nouveau sens de ce meurtre dans une société actuelle :

"L'infanticide est lui-même soumis à des
interprétations nouvelles chez les modernes : un
adoucissement de la mort apparaît [...]. A la violence
virile des Médée antiques se substitue une image
moins sauvage où la féminité et les sentiments
maternels l'emportent. " 1

C'est une Médée plus maternelle, elle préfère ici les voir mourir plutôt que de les voir souffrir. Les propos d'Ariane Eissen, nous pouvons dire qu'en offrant ses enfants comme victimes expiatoires aux mânes de son frère, Médée se purifie de ses crimes passés. Ainsi redevient-elle la jeune fille qui ne connaissait pas encore Jason. ${ }^{2}$

Jahnn tente de donner une explication valable qui tend à ne pas culpabiliser Médée. Ce sont la passion et l'amour d'une mère qui conduisent à ce désastre.

\section{La fatalité de la femme Médée}

Le thème de la femme considérée comme une figure essentielle de la littérature par rapport à sa fonction sociale dominante. Ainsi, Médée l'une des figures mythiques destructrices (femme fatale). La femme d'après Jean Girodet:

1. Duarte Mimoso Ruiz, op. cité, p. 205.

2. Ariane Eissein, « Médée la magicienne » in Les mythes grecs, Paris, Belin, 1993, p. 89.

3. Jean Girodet, Dictionnaire de la langue française, Paris, Bordas, 1985, pp. 1198-1199. 
"Désigne l'être qui a tous les caractères généralement attribués à la descendance d'Eve : la séduction, l'altruisme, la douceur lui sont accordés par opposition à l'homme.. ${ }^{3}$

Le terme "fatale " désigne ce qui a été marqué par le destin, ce qui doit arriver inévitablement.

Médée présente donc deux visages superposés et contradictoires : celui de la victime et du bourreau. Elle pourrait être considérée comme une femme fatale. Toutes amoureuses, une mère tendre mais aussi une femme destructrice.

\section{La complexité de Médée réside dans cette dualité}

Médée s'est également autodétruite à travers l'infanticide qu'elle a commis. En tuant ses enfants pour blesser Jason, Médée connaît aussi la souffrance comme une mère séparée de ses enfants. Nous pouvons dire que Médée est effectivement une femme fatale.

\section{Médée: une réception contemporaine}

Médée reflète la révolte de la femme contemporaine:

Médée de Jahnn est marquée par la modernisation: un nouveau statut de Médée. En effet, Médée est comme une africaine en révolte contre les actions de Jason et de la société corinthienne. Jahnn traduit les maux sociaux qui minent l'époque actuelle tels que: le rejet de l'étranger, la marginalité de la femme et les problèmes politiques.

Chez Jahnn Médée est une africaine à la peau très noire (une Médée d'une laideur repoussante). Elle s'est laissée vieillir pour Jason et pour ses enfants afin que ces derniers gardent leur beauté et leur jeunesse éternellement. Malgré ses efforts, elle subit le mal de discrimination élémentaire à cause de la couleur de sa peau. 
Jahnn a choisi de relire le mythe de Médée à la lumière de son époque. Il l'a réécrit en fonction des problèmes contemporains qui touchent son pays, le monde entier.

\section{CHAPITRE III: MÉDÉE DE JEAN ANOUILH}

L'histoire d'une passion

\section{Médée}

Le monde est Médée pour toi, à jamais.

Jason

Le monde a-t-il donc toujours été Jason pour toi؟

\section{Médée}

\section{Oui! $^{1}$}

La pièce Médée de Jean Anouilh, tragédie écrite en 1946(publiée dans les Nouvelles pièces noires), reprend les éléments du mythe pour leur donner un sens nouveau. Jean Anouilh confronte Médée la bohémienne à l'univers de l'ordre incarné par Jason.

" Médée est avant tout une femme trompée par son amant: " Anouilh est intéressé moins par l'infanticide de Médée que par le divorce des deux héros" ${ }^{2}$

Jean Anouilh est un écrivain et dramaturge français, né 1910 à Bordeaux (Gironde) et mort 1987 à Lausanne (Suisse).

"Son œuvre théâtrale commencée en 1932. Elle est constituée de nombreuses comédies et d'œuvres dramatique ou tragique comme Antigone. ${ }^{3}{ }^{3}$

1. Anouilh, Jean, Médée, Paris, La table ronde, 1961, p. 380.

2. Eissen, Ariane, Les mythes grecs, op. cit., p. 95.

3. https://fr.wikipedia.org/wiki/Jean_Anouilh 
La pièce s'inspire du mythe de Médée, dix ans après avoir trahi son père et tué son frère à cause de son amour pour Jason et la conquête de la Toison d'or. Alors, Jason s'éloigne d'elle et s'apprête à épouser Créüse, refusant le bonheur, Médée va continuer à semer le feu de vengeance.

L'œuvre de Jean Anouilh garde les éléments du mythe de Médée. L'action est concentrée autour de quelques personnages, Médée, Jason, Créon, et la Nourrice de Médée. En fin, Médée brûlante dans les flammes, Jason empêche toute intervention. Chez Anouilh, le mythe est modernisé: Médée vit dans une roulotte. De même, Anouilh analyse la complexité des rapports hommes-femmes.

Une tragédie brûlante et déchirante où les désirs absolus ne connaissent pas de limites.

La pièce d'Anouilh trace une image merveilleuse de la résistance. C'est une écriture très intéressante:

"Médée libre de dire non. Héroïne résistante." ${ }^{1}$ "Aussi, Chez Anouilh, les dieux laissent leurs places aux humains. Les problématiques amoureuses sont plus accessibles. Sa pièce commence, à la différence des ancêtres, au moment où Médée apprend la trahison de Jason pour une autre. $\|^{2}$

\section{De Médée contemporain renaît la Médée mythologique}

\section{Médée l'amoureuse}

Dès le début de la pièce, le personnage de Médée explique à la nourrice pourquoi elles sont fuites de leur terre natale, la Colchide:

1. https://femmes-de-lettres.com/2015/02/16/les-reecritures-de-medee411-medee-de-jean-anouilh/

2. Ibid. 
" On est parties parce que j'aimais Jason, parce que j'avais volé pour lui mon père, parce que j'avais tué mon frère pour lui! " ${ }^{1}$

Médée a accepté de changer de statut social en se liant avec Jason, passant du statut de princesse à celui de voleuse et d'étrangère, abandonnant ses richesses pour vivre dans la pauvreté et la misère:

" Tu avais un palais aux murs d'or et maintenant nous sommes là ، accroupies comme deux mendiantes, devant ce feu qui s'éteint toujours» 2

Malgré ce mode de la fuite et de l'errance، Médée ne regrette pas sa vie, alors qu'elle était princesse. Ce qui compte pour elle, c'est de partager sa vie avec Jason. Ainsi lorsque la nourrice déclare:

"Chassées, battues, méprisées, sans pays, sans maison $)^{3}$

Anouilh réussit à analyser profondément les passions les êtres humains. Dans cette version, Médée est sûrement malade d'Amour pour Jason. Pour cet amour elle tue et devient donc une criminelle

Elle continuera dans cette voie destructrice jusqu'à la fin.

C'est une Médée du vingtième siècle qui se trouve dans l'œuvre d'Anouilh. Les personnages sont moins héros, plus humains. Pas de Dieux. La destinée de Médée n'est pas la question de cette version.

Elle avoue à la nourrice que son départ de Colchide et le meurtre de son frère sont les preuves de l'amour pour Jason.

1. Anouilh, Jean, Médée, op. cit, p. 356.

2. Ibid.

3. Ibid, p. 358. 
" Oui " et " non " souvent, s'opposent de l'acceptation (Jason, Créon et la nourrice) et du refus (Médée, seule).

Médée, refuse le bonheur :

"Quelque chose se trouve dans moi qui dit non au bonheur. " 1

La lumière tragique naît du malentendu entre un amour fou et une lucidité aveuglante. La cohabitation de la lucidité et de l'aveuglement fait du temps tragique un moment singulier.

\section{La sensualité et la sexualité}

La sensualité et la sexualité jouent un rôle prépondérant dans la version des contemporains.

Une grande partie de l'amour de Médée est justifiée dans l'œuvre d'Anouilh par son désir sexuel pour Jason:

«Je l'attendais tout le jour,... »"

Contrairement à la Médée d'Euripide, la Médée d'Anouilh s'agit d'un violent désir:

" Ô soleil, si c'est vrai que je viens de toi, pourquoi m'as-tu faite une fille ? Pourquoi ces

1. Ibid.p.16.

2. Ibid, p. 362. 
seins? N'aurait-il pas été beau le garçon Médée? ${ }^{1}$

Médée, chez J. Anouilh, va encore plus loin dans sa révolte contre l'homme:

«Elle méprise sa dépendance vis-à-vis de Jason, tout en reconnaissant implicitement, qu'elle ne peut s'en passern. ${ }^{2}$

Contrairement à l'abandon de Jason, Anouilh ajoute un nouvel élément au mythe en écrivant que c'est Médée qui a été infidèle:

"Mais cette couche où tu nous prétends liés à jamais, qui l'a désertée la première? Qui, la première a accepté d'autres mains sur sa peau, le poids d'un autre homme sur son ventre? ${ }^{3}{ }^{3}$

La révolte de Médée se situe dans un contexte social où la liberté sexuelle de l'homme et de la femme s'affirme avec autant de force. Le problème des enfants et de la famille tend à passer au deuxième plan, au profit de considérations sur le couple.

La Médée d'Anouilh agit ainsi avec plus de liberté que la Médée d'Euripide

3. L'abandon, la crainte et la solitude de Médée

Le désespoir d'une personne qui se retrouve seul, privé de l'être aimé, sans lequel la vie paraît vide, dénuée de tout sens. C'est le cas de Médée qui se sent triste en raison de

1. Ibid, p. 363.

2. Mimoso-Ruiz, Duarte, Médée antique et moderne, Aspects rituels et socio-politiques d'un mythe, Paris, Ophrys, 1982,169 p.

3. Anouilh, Jean, Médée, op. cit, p. 380. 
l'absence de Jason. À partir du moment où Médée apprend que Jason l'abandonne pour une autre femme, l'amour qu'elle lui porte semble se transformer en haine. Toute cette haine n'est qu'un long et douloureux cri d'amour qui démontre la souffrance profonde de Médée .

\section{Elle est prête à changer pour le garder et qu'elle se tue par amour pour lui.}

Tous ces sacrifices, consentis pour Jason, ne trouvent aucune récompense auprès de lui. Jason s'éloigne d'elle. Elle cherche à comprendre les raisons de l'attitude subite de son mari qui lui avait pourtant juré fidélité.

En plus de cet abandon, elle constate que Jason ne s'oppose pas à la décision du roi Créon, qui veut son expulsion ainsi que celle de ses enfants. Son désespoir n'est pas uniquement fondé sur la trahison de son mari mais également sur son rejet par les Corinthiens. Elle va provoquer une solitude profonde. Médée se retrouve seule face à son destin. Elle est désormais la seule à payer ses crimes.

Créon vient lui annoncer qu'elle va devoir quitter Corinthe. La Colchidienne a en effet des antécédents de mensonges, de vols et de meurtres. Si Médée refuse de partir, Créon va la livrer aux fils de Pélias qui aimeraient bien venger l'assassinat de leur père.

Selon Créon, Jason, contrairement à elle, est innocent. De plus, Jason est un fils de Corinthe tandis que Médée n'est qu'une barbare. Tout comme chez Euripide, Médée incarne l'Autre, l'étrangère à la fois barbare et menaçante, comme la nourrice elle-même le souligne: 
« On n'est pas d'ici " ${ }^{1}$

Contrairement à la pièce d'Euripide la Médée d'Anouilh contient un discours politique important. Chez Anouilh:

" la transposition opérée dans le mythe est donc une satire symbolique des conditions de la période contemporaine, lesquelles s'expriment dans les guerres, les discriminations de toutes sortes, le fascisme, le rejet et le refus de l'Autre ret la violence ". ${ }^{2}$

Chez Anouilh ، Médée n'accepte pas de perdre son identité en se soumettant aux Corinthiens. Elle représenterait les femmes qui protestent contre un système pour retrouver leur dignité.

Comme dans la version d'Euripide, cette princesse est contrainte une fois de plus à l'exil. Mais, cette fois-ci, Médée est sommée de s'en aller seule sans Jason.

Il enlève ainsi les enfants à la mère qui se retrouve toute seule. Dépouillée, humiliée et avilie par Jason, Médée devient jalouse, d'une jalousie aveugle et maladive.

\section{La jalousie aveugle et maladive de Médée}

Médée refuse que Jason commence une vie avec une autre. Elle devient alors intransigeante et impatiente. Les cris et les pleurs montrent son désespoir face à cette trahison:

1. Mimosa-Ruiz, Duarte, Médée antique et moderne, Aspects rituels et socio-politiques d'un mythe, op. cit, p. 191.

2. Koua, Viviane, Médée figure contemporaine de l'interculturalité (Thèse de doctorat) - Université de Limoges et Université de Cocody), 2006 [En ligne], p. 13. 
«Nourrice, je suis grosse ce soir. J'ai mal et j'ai peur comme lorsque tu $\mathrm{m}$ 'aidais à me tirer un petit de mon ventre... $C^{\prime}$ est quelque chose qui dit non au bonheur. ${ }^{1}$

De la haine, la jalousie ici personnifiée. Médée a été bien animée par la haine et la jalousie:

"O ma haine ! Comme tu es douce, comme tu sens bon. Petite fille noire, toi que je n'ai plus qu'au monde à aimer. $\|^{2}$

La jalousie sera le déclencheur de la pensée vindicative. En effet, le qualificatif " noir " peut évoquer ici le malheur, le désastre, qui trouve leur origine dans la souffrance de Médée.

Elle a tué pour Jason et pour la première fois, elle le fera pour elle:

" Je t'ai suivi dans le sang et dans le crime, il va me falloir du sang et un crime pour te quitter ${ }^{3}$

La violence est omniprésente chez Médée. Si Jason veut refaire sa vie avec une autre femme, elle va tuer ses enfants:

" Ô mal! Grande bête vivante qui rampe sur moi et me lèche, prends-moi. Je suis à toi cette nuit, je suis ta femme.» 4

Selon les ordres de Médée, la nourrice doit réveiller les enfants et aller chercher le coffre noir qui contient le cadeau de noces à Créüse. Le coffre renferme un voile d'or et un diadème empoisonnés. Son cadeau de noces amené par les enfants cause la mort de la princesse et du roi:

1. Jean Anouilh, op.cit. p. 359.

2. Ibid. p. 362.

3. Ibid. p. 365.

4. Ibid. p. 393. 
« Tout est perdu! La royauté, l'État sont tombés. Le roi et sa fille sont morts ! " ${ }^{1}$

\section{Le cadeau fatal : le meurtre de Créüse et de Créon}

La magie de Médée est très peu soulignée dans la version d'Anouilh. C'est une femme de chair avant tout. Anouilh n'insiste donc pas sur le fait qu'elle est magicienne mais plutôt sur sa condition humaine.

Le porteur de nouvelles suggère à Médée de fuir, car les hommes savent qu'elle est responsable de la mort de leur roi et ils accourent vers la roulotte munis de bâtons et de couteaux afin de se venger.

\section{L'infanticide et la suicide de Médée}

Médée d'Anouilh refuse de continuer à vivre dans ce monde de trahison, en préférant la suicide et donner un deuxième désespoir à Jason: au début le meurtre de leurs enfants.

\section{"L'infanticide résulte de la trahison dans l'amour, aussi, il est lié, d'autre part au destin.." ${ }^{2}$}

Malheureuse, Médée se transforme en une sorte de meurtrière. L'amour se transforme en haine destructrice. Médée ne se contente pas uniquement de la mort de Créüse et de Créon. Cette fois ' l'objet de sa fureur meurtrière se trouvent être ses propres enfants.

Alors que la nourrice s'occupe de mettre des fagots sous la roulotte pour préparer un feu, les enfants viennent retrouver leur mère. Médée n'est ici pas déchirée par son amour pour ses enfants. C'est une amante et non une mère que peint Anouilh:

1. Ibid. p. 394.

2. http://france.tabrizu.ac.ir/article_4453_22c764291a511eac1f2354f8aeb8 c4af.pdf 
"Jason ! Voilà ta famille, tendrement unie. Quand tu souffriras, pense qu'il y a eu une Médée exigeante et pure autrefois. ${ }^{1}$

Médée rentre dans la roulotte où elle égorge les enfants. Des flammes jaillissent de partout. Jason ordonne à ses hommes d'éteindre le feu.

C'est alors que Médée annonce à Jason que ses enfants sont morts :

" Ils sont morts égorgés tous les deux, et avant même que tu aies pu faire un pas, ce même fer va me frapper. Désormais j'ai recouvré mon sceptre; mon frère ، mon père et la toison et j'ai retrouvé ma patrie et la virginité que tu $m$ 'avais donné !»

Médée se frappe et s'écroule dans les flammes. La mort de Médée est une variante plus importante de la pièce d'Anouilh puisque, chez Euripide, Médée fuit pour retrouver Égée sur un char solaire. Aussi, Anouilh laisse les enfants et Médée brûler dans la roulotte alors que chez Euripide. Chez Anouilh, Médée ne semble éprouver aucune douleur face à l'exécution des enfants.

Le suicide puis l'infanticide semblent être les seules voies pour Médée pour échapper à sa condition.

Elle est une mère malgré ses violences d'attitudes: Médée ne veut pas que ses enfants être tués par corinthiens et elle cherche à pousser sa vengeance à l'extrême pour plonger Jason traître dans le désespoir. Par la mort de ses enfants, elle se libèrera.

La pièce se termine alors que la roulotte brûle. C'est le retour à l'ordre. La mort de Médée permet à Jason de régner sur Corinthe:

1. Jean Anouilh, op.cit. p. 396.

2. Ibid. p. 397. 
" Jason est donc, sans Médée, ne serait devenu le ravisseur de la Toison d'or, mais qu'avec elle, il ne peut être un chef et un héros.

C'est pourquoi, après la mort des enfants, et la suicide de Médée, le rideau tombe sur les appels à l'ordre de Jason, qui prétend régner». ${ }^{1}$

Chez Anouilh, l'accent est mis sur la relation entre Médée et Jason, Le meurtre des enfants devient alors secondaire. Cette fois, nous quitterons la Grèce afin de voir comment l'auteure réactualise le mythe dans le $X X^{e}$ siècle.

Médée de Anouilh est marquée du sceau de la modernisation. II confère au personnage de Médée un nouveau statut. En effet, Médée est présentée comme une gitane en révolte contre les actions de Jason et de la société corinthienne. Sa préoccupation majeure en réécrivant le mythe est de traduire à sa manière les maux sociaux qui minent encore l'époque actuelle tels que: la haine, le rejet de l'étranger, la marginalité de la femme et les problèmes politiques.

1. Eissen, Ariane, Les mythes grecs, op. cit., p.95. 


\section{CONCLUSION}

Après avoir analysé le personnage de Médée, afin de voir de quelle façon il varie, de l'antiquité au temps contemporain, d'un auteur à l'autre, on peut constater que chaque auteur ajoute des variantes propres au contexte de son œuvre.

Ce mythe connaît une grande popularité chez les auteurs modernes qui cherchent à représenter les cultures différentes à travers le personnage de Médée. Du même, les auteurs, par Médée, peuvent exprimer les problèmes sociaux de leurs époques, les conditions féminines et l'injustice politique.

Dans le mythe antique, Médée se révolte contre l'abus de pouvoir de roi Créon. Mais chez Jahnn et Anouilh :

"Elle représente la farouche opposante à tous les régimes totalitaires qui s'octroient le droit de décider à la place des autres. Dès lors, le combat de Médée constitue la lutte contre l'inégalité et |'injustice sociale» ${ }^{1}$

Ainsi, Médée a réussi à être une figure féminine et mythique de l'iniquité au temps contemporain.

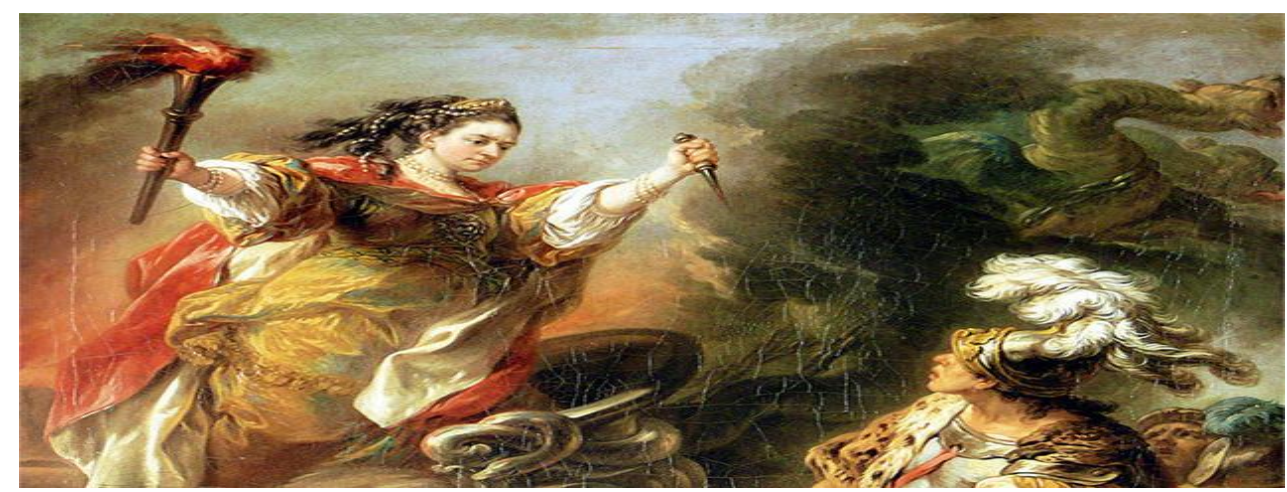

1. Koua, Viviane, Médée figure contemporaine de l'interculturalité, op. cit., p. 329. 


\section{BIBLIOGRAPHIE}

- Cuvres étudiées:

1. Anouilh, Jean, Médée, Éditions de la Table ronde, 1947. Création de la pièce : 1946.

2. Euripide, Médée, Paris, Les Belles lettres, 1965.

3. Jahnn, Hans Henny, Médée [1963], Paris, José Corti, 1988. Jahnn, Hans Henny, Médée, traduit par Huguette et René Radrizzani, Paris, éd. José Corti, 1998.

\section{- CEuvres littéraires et dictionnaires:}

1. Alain, Moreau, "Médée ", Dictionnaire des mythes féminins, sous la direction de Pierre Brunei, Monaco, Du Rocher, 2002.

2. Brunel, Pierre, Claude Pichois, André-Michel Rousseau, Qu'est-ce que la littérature comparée ? Paris : Armand Colin, 1996.

3. Depaulis, Alain, Le Complexe de Médée : quand une mère prive le père de ses enfants, Bruxelles, De Boeck et Larcier s. a. 2003.

4. Det, Jean Giro, Dictionnaire de la langue française, Paris, Bordas, 1985.

5. Eissein, Ariane, "Médée la magicienne " in Les mythes grecs, Paris, Belin, 1993.

6. Eliade, Mircea, Aspect du mythe, Paris, Gallimard, 1966.

7. Girodet, Jean, Dictionnaire de la langue française, Paris, Bordas, 1985.

8. Grimal, Pierre, Dictionnaire de la mythologie grecque et romaine, Paris, PUF, 1951.

9. Jahnn, Hans Henny, Texte français René Radrizzani, Extrait de "Les Légendes de Médée et de savie»,1927.

10. Koua, Viviane, Médée figure contemporaine de l'interculturalité (Thèse de doctorat) -Université de Limoges et Université de Cocody), 2006 [En ligne]. http://www.unilim.fr/theses/2006/lettres|2006limo0006/koua _ v.pdf (Page consultée le 10avril 2007). 
11. Mimoso-Ruiz, Duarte, Médée antique et moderne, Aspects rituels et socio-politiques d'un mythe, Paris, Ophrys, 1982.

12. Ruiz, Duarte Mimoso, Avatars modernes du mythe antique dans deux Médée Africaines, Strasbourg : 1980.

\section{CITOGRAPHIE}

1. https://litterature.savoir.fr/mythe-litteraire-definition

2. http://www.mythologie.ca/heros/medee.html

3. https://www.etudes-litteraires.com/forum/topic44268-maxrouquette-medee-scene-21.html

4. https://www.chapitre.com/BOOK/jahnn-hanshenny/medee,1203491.aspx\#product-details-and-summary

5. http://www.fabriktheatre.com/spectacles/medee-de-hanshenny- Jahnn/

6. https://femmes-de-lettres.com/2015/02/21/les-reecrituresde-medee-medee-de-hans-henny-jahnn/

7. http://next.liberation.fr/livres/1998/10/29/espece-de-medeeune-medee-de-1926-violente-et-raciale-par-hans-hennyjahnn-allemand-a-la-sensualite- 249333

8. https://fr.wikipedia.org/wiki/Jean_Anouilh

9. http://france.tabrizu.ac.ir/article_4453_22c764291a511eac1f2 354f8aeb8c4af.pdf

10. (https://femmes-de-lettres.com/2015/02/21/les-reecrituresde-medee-medee-de-hans-henny-jahnn/ 


\section{Table des matières}

\begin{tabular}{|c|c|c|}
\hline \multicolumn{2}{|c|}{ Titre } & \multirow{2}{*}{$\begin{array}{l}\text { bage } \\
2\end{array}$} \\
\hline 1 & INTRODU CION & \\
\hline 2 & PROBLÉMATIQUE DE L'ÉTUDE & 2 \\
\hline 3 & CORPUS DE L'ÉTUDE & 4 \\
\hline 4 & MÉTHODE D'ANALYSE & 5 \\
\hline 5 & $\begin{array}{l}\text { CHAPITRE I: } \\
\text { SOURCES ET RÉSUME DU MYTHE DE MÉDÉÉ }\end{array}$ & 6 \\
\hline 6 & Le personnage de Médée chez Euripide & 7 \\
\hline 7 & $\begin{array}{l}\text { CHAPITRE II: } \\
\text { MÉDÉE Hans Henny Jahnn }\end{array}$ & 10 \\
\hline 8 & $\begin{array}{l}\text { Pourquoi l'être de Médée se mue pour se conjuguer à } \\
\text { l'universel }\end{array}$ & 15 \\
\hline 9 & Médée: une réception contemporaine & 19 \\
\hline 10 & $\begin{array}{l}\text { CHAPITRE III: } \\
\text { MÉDÉE DE JEAN ANOUILH }\end{array}$ & 20 \\
\hline 11 & De Médée contemporain renaît la Médée mythologique & 21 \\
\hline 12 & CONCLUSION & 32 \\
\hline 13 & BIBLIOGRAPHIE & 33 \\
\hline 14 & TABLE DES MATIÈRES & 35 \\
\hline
\end{tabular}

